\title{
Melanoma Detection in Dermoscopic Images using Global and Local Feature Extraction
}

\author{
JC Kavitha ${ }^{1}$, Suruliandi $\mathrm{A}^{2}$ and Nagarajan $\mathrm{D}^{3}$ \\ ${ }^{1,2}$ Manonmaniam Sundaranar University, Tirunelveli, Tamil Nadu, India \\ ${ }^{3}$ Debra Tabor University, Ethiopia \\ 1jck_kavitha@yahoo.co.in, ${ }^{2}$ suruliandi@yahoo.com, ${ }^{3}$ dnrmsu2002@yahoo.com
}

\begin{abstract}
Feature plays an important role in the processing of medical images. The different features of an image include color, texture, shape or domain specific features. Texture is considered as one of the important feature of an image. In this paper, the global and local texture feature extraction is done using different algorithms. The global texture features for an image such as energy, entropy, homogeneity, correlation, contrast, dissimilarity, maximum probability are computed using Gray level co-occurrence matrix (GLCM). The local texture features for an image is extracted using a texture feature descriptor named Speeded Up Robust Features (SURF). The performance of feature extraction is based on the classification results. The process of classification is done using Support vector machine (SVM) and KNN classifier. The performance is evaluated on the basis of different metrics namely sensitivity, specificity, accuracy, precision and F1 score. The experimental result shows that the local texture feature extracted using SURF performs best when compared to global feature extraction (GLCM) and also with other descriptors such as Scale Invariant Feature Transform (SIFT). The SURF local feature descriptor along with SVM - RBF classifier provides better classification accuracy.
\end{abstract}

Keywords: Gray level co-occurrence matrix (GLCM), Speeded Up Robust Features (SURF), Scale Invariant Feature Transform (SIFT), Support vector machine (SVM), KNearest Neighbor $(K N N)$

\section{Introduction}

Melanoma is considered as one of the most malignant, metastatic and dangerous form of skin cancer. It is responsible for the majority of deaths related to skin cancer. The curability and survival rate of a patient can be increased if melanoma can be detected at an earlier stage [1]. The skin lesions are diagnosed by dermatologists using a technique known as dermoscopy. This is a non-invasive procedure used for the in vivo observation of skin lesions. The skin cancers are classified into three types namely melanoma, basal cell carcinoma (BCC) and squamous cell carcinoma (SCC). Melanoma is a condition or a disorder that affects the melanocyte cells thereby impeding the synthesis of melanin [2]. The disorder is characterized by the development of lesions in the skin and it varies in shape, size, color and texture. The texture features are considered to be important in the detection of melanoma. The texture is characterized by the spatial distribution of pixels in the neighborhood of an image. The spatial dependence of gray levels is represented by a two dimensional matrix known as GLCM and it is used for global texture analysis of an image. The GLCM matrix specifies the texture of an image that how often the pairs of pixels with specific values occur in an image. The statistical measure is then extracted from the GLCM matrix. The textural features represent the spatial distribution of gray tonal variations within a specified area. In images, the neighboring pixel is correlated and spatial values are obtained by the redundancy between the neighboring pixel values [3]. The local texture features are computed using SURF descriptor. In SURF, there are three 
steps namely interest point detection, interest points description and feature descriptor matching. The interest points are detected using the factor repeatability and a descriptor vector is generated based on these interest points. The descriptor vector is obtained by summing the Haar wavelet responses (HWR). The feature vectors are matched against the neighbor using Euclidean distance [5].

Support Vector Machine (SVM) [17] is a supervised learning model used for classification and regression analysis. It analyses the image and data patterns used in image processing. The advantage of support vector machine is that it works effectively in high dimensional spaces It is also memory efficient because of the training points used in the decision function called support vectors and different kernel functions can be used for decision function.

\subsection{Related Works}

There are several systems for the detection of melanoma from dermoscopic images. The classification of skin lesion is done using both global and local feature extraction techniques. In both the methods of global and local texture feature extraction, the process of segmentation is done using simple adaptive thresholding algorithm. In global method, the texture features are extracted using GLCM matrix in four different orientation angles. In local method, the texture features are extracted using SURF. The classification is done using SVM-RBF and KNN classifier for the detection of melanoma. The experimental result shows that SVM-RBF provides better classification accuracy when compared to $\mathrm{KNN}$ classifier for texture feature extraction.

In this paper, the textural features are considered for classifying the image. These textural features are calculated in the spatial domain and a set of gray tone spatial dependency matrix was computed. The textural features are computed using GLCM matrix in four different orientation angles. The textural features are based on the statistics that describes how the gray tone appears in a spatial relationship to another [3]. In ELM or dermoscopic images, the malignancy of a lesion is anlyzed using an automated method. In this proposed method, the segmentation process is done using thresholding operation combined with color clustering. Dynamic thresholding provides good segmentation results. A set of features namely shape and color is used to describe the malignancy of a lesion. Sequential forward selection algorithm is used for feature selection. The classification is done using KNN classifier and it classifies the dermoscopic image into benign or malignant [6].

A novel method was proposed to classify the dermoscopic images. In this method, automatic border detection was done using JSEG algorithm to extract the skin lesion. The preprocessing of an image is done using color median filter and color reduction is done using variance based quantization. Skin lesions are characterized using shape, color and texture features. Shape features include area, aspect ratio, asymmetry and compactness. Color features are calculated in color spaces namely RGB, HSV and LUV. The texture features are extracted using GLCM in four different orientation angles. In order to increase the prediction accuracy and to reduce the number of irrelevant and noisy patterns, the process of feature selection is performed using filters and wrappers. The classification of images is done using SVM classifier [7],[15]. In this automated approach of skin lesion identification [8], the skin lesions are extracted using segmentation and border detection. The segmentation process is done using laplacian filter and border detection using zero crossing algorithm. The texture features are extracted by computing the variables mean, contrast, entropy and fractality. The mean of the color space RGB inside and outside the lesion is calculated to detect whether the person is affected by melanoma or not. ANN and Feed Forward ANN classifier is used to classify beningn and melanoma from the dermoscopic images.

Lowe [4] proposed a local texture feature descriptor SIFT which is invariant to image scale, rotation and partially invariant to illumination. The steps in SIFT icludes scale 
space extrema detection, keypoint localization, orientation assignment and key point description. It computes a histogram of locally oriented gradients around the interest point. The size of the SIFT descriptor depends on the orientation histograms and the number of orientation bins in each region. The performance of the descriptor depends on the accuracy of interest point and region detection.

Herbert Bay, tuytelars [5] proposed that SURF outperforms other descriptors in terms of distinctiveness, robustness, repeatability and speed. SURF combines the gradient information with the local pixels whereas SIFT depends on the orientation of individual gradients. SURF is divided into three steps namely interest point detection, interest point description and feature vector matching. Since SURF uses a Fast-Hessian based detector, it is fast and repeatable when compared to SIFT. Schimd et al. [9] compared the performance of local interest point descriptors. The descriptors are evaluated based on ROC (Receiver operating characteristics) for a query image with respect to false positive rate. The evaluation is carried out for various image transformations such as scale, rotation and illumination changes. The performance of the descriptor depends on the accuracy of interest point detection.

\subsection{Motivation and Justification}

There were many texture feature extraction methods explained above and of which SURF descriptor is widely used in texture feature extraction of images [5][9]. The skin regions affected by melanoma are predominated by texture. GLCM extracts the texture features and if the dimensionality of the feature vector is high, feature selection is necessary. Therefore, it is essential to select the relevant features from the high dimensional feature vector and it can be done through feature descriptors such as SIFT and SURF [11]. SVM is used for the classification of melanoma from dermoscopic images.

In SIFT and SURF, the interest points were selected and based on these interest points the descriptor vector is generated. The features extracted using the texture feature descriptors are given as an input to the SVM and KNN classifier. The computational speed of SURF is very high when compared to other descriptors in the classification of melanoma. Justified by these facts, SURF is used for local texture feature extraction and it also invariant to scale, rotation and illumination changes.

\subsection{Outline of the Proposed Approach}

The proposed system deals with global and local texture feature extraction using GLCM and SURF [12]. In this, the skin lesion is extracted from the dermoscopic image using the process of segmentation by Otsu's adaptive thresholding method. The global texture features are extracted using GLCM in different orientation angles. The local texture features are extracted using SURF feature descriptor. The classification of melanoma from dermoscopic images is done using SVM and KNN classifier. The proposed approach for texture feature extraction is shown in the below Figure1. 


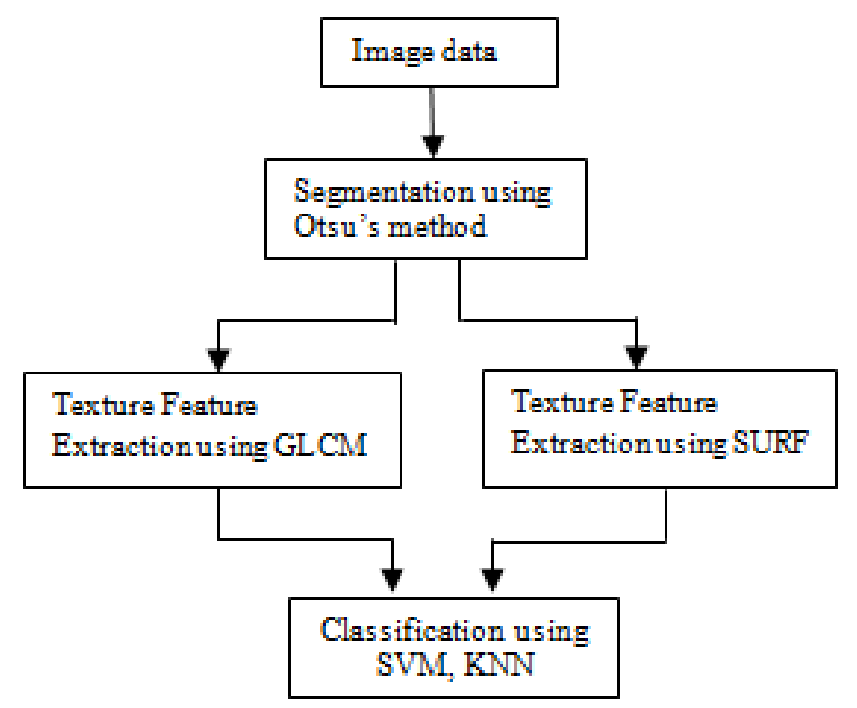

Figure 1. Feature Extraction and Classification

\subsection{Organisation of the Paper}

The Section 2 presents the overview of texture feature extraction using GLCM and SURF feature descriptor. The Section 3 describes the classification algorithm using SVM and KNN. The Section 4 presents a procedure for feature extraction and classification. The Section 5 presents a detailed description of the experiments conducted on dermoscopic images. The performance of the proposed system is also analyzed in this section. The final section presents the results of the experiments and thus gives the conclusion.

\section{Texture Feature Extraction}

\subsection{Gray Level Co-Occurrence Matrix (GLCM)}

In statistical texture analysis, the texture features are obtained from the statistical distribution of intensities at particular position relative to each other in an image. The texture statistics are divided into first order, second order and higher order statistics. First order texture measures are statistics calculated from original image and do not consider pixel neighbor relationships. The Gray level Co-occurence matrix (GLCM) is a method of extracting second order statistical texture features [3]. GLCM considers the relation between two pixels at a time, called reference pixel and a neighbor pixel. A GLCM is a matrix where the number of rows and columns are equal to the number of gray levels $G$ in an image. The matrix element $P(i, j \mid d, \theta)$ contains the second order statistical probability values for changes between gray levels $i$ and $j$ at a particular displacement distance $d$ and particular angle $\theta$. Element $[i, j]$ of the matrix is generated by counting the number of times a pixel with value $i$ is adjacent to a pixel with value $j$. Each entry is the probability that a pixel with value $i$ is found adjacent to a pixel of value $j$. The different textural features is computed using GLCM matrix in four different orientation angles.

\subsection{Speeded UP Robust Features (SURF)}

SURF is a scale and rotation invariant detector and descriptor. SURF outperforms other descriptors in terms of repeatability, distinctiveness, robustness and speed [4]. It is divided into three steps namely interest point detection, interest point description and feature descriptor matching. In an image, the interest points are selected at different locations such as blobs, corners and T-junctions. The most important feature of interest 
point detector is repeatability. It verifies whether the same interest points are detected under different viewing conditions. The interest points are detected using Hessian matrix. This involves scale space construction and the interest points are determined based on approximating the convolution of Gaussian second order partial derivatives using box type filters. It has been proved that Hessian based detectors are more stable and repeatable $[10,11]$. It reduces the computation time and hence called Fast-Hessian detector. Given a point $\mathrm{X}=(\mathrm{x}, \mathrm{y})$ in an image I. It has been shown that SURF is fast since it uses FastHessian Detector when compared to SIFT that uses DoG $[4,16]$. The Hessian matrix $\mathrm{H}(\mathrm{x}, \sigma)$ in $\mathrm{x}$ at scale $\sigma$ is defined as

$$
H(x, \sigma)=\left[\begin{array}{ll}
L_{x x}(x, \sigma) & L_{x y}(x, \sigma) \\
L_{x y}(x, \sigma) & L_{y y}(x, \sigma)
\end{array}\right]
$$

where $\mathrm{L}_{\mathrm{xx}}(\mathrm{x}, \sigma)$ is the convolution of the gaussian second order derivative with the image $\mathrm{I}$ in point $\mathrm{X}$. It is given by

$$
\begin{gathered}
L_{x x}(x, \sigma)=I(x) * \frac{\partial^{2}}{\partial x^{2}} g(\sigma) \\
\mathrm{L}_{x y}(\mathrm{x}, \sigma) \text { is given by } L_{x y}(x, \sigma)=I(x) * \frac{\partial^{2}}{\partial x y} g(\sigma)
\end{gathered}
$$

A descriptor vector corresponding to an interest point is generated and it is obtained by selecting a circular neighbourhood region around the interest point and Haar wavelet responses (HWR) are calculated both in horizontal and vertical direction. The orientation is calculated by adding the horizontal and vertical responses and this yields a new vector. Since SURF is invariant to rotation, a main orientation angle is fixed around the interest points. Once the main orientation angle is assigned, the descriptor is extracted by constructing a square region around the interest point and it is oriented along the main orientation angle. The selected square region is split up into $4 \times 4$ sub regions. For each sub region, the HWR in horizontal and vertical directions are calculated and are summed up over each sub region and form a set of entries to the four dimensional feature vectors. The descriptor vectors are matched between different images. The matching of images is done using Euclidean distance. If the Euclidean distance is closer to the nearest neighbour, then a matching pair is detected. It has been shown that SURF is fast since it uses Fast-Hessian Detector when compared to SIFT that uses DoG $[5,16]$.

\section{Classification}

\subsection{Support Vector Machine (SVM) Classifier}

Support vector machines [17] are supervised learning models with associated learning algorithms that analyze the data and recognize patterns used for classification. Support vector machine constructs a hyper-plane or set of hyper-planes in a high or infinitedimensional space. A good separation is achieved by the hyper-plane which has the largest distance to the nearest training-data point of any class. In general, larger the margin, lower the generalization error of the classifier. It is mainly applicable for solving the binary problems.

\subsection{K- Nearest Neighbour Classifier (KNN)}

$\mathrm{KNN}$ is a non-parametric method used for classification and regression [18]. The input consists of k-closest training examples in the feature space. The training part of the algorithm consists of storing the feature vectors and class labels of the training samples. In classification phase, it is classified depending on the value of $\mathrm{k}$ among the training samples closest to the query point. The Euclidean distance is used to measure the distance 
between the nearest training samples.

\section{Procedure for Feature Extraction \& Classification}

Training Phase:

- Read the testing data set from the local repository.

- Segment the lesion region from an image.

- Compute the global texture feature from the segmented region using GLCM.

- Compute the local texture feature using SURF.

- Pass it as an input to SVM and KNN classifier.

- Classify it into melanoma or non-melanoma.

Testing Phase:

- Read the training dataset from the local repository.

- Apply the training model to the testing data set for prediction accuracy.

- Classify it into melanoma and non-melanoma.

\section{Experiments and Results}

\subsection{Experimental Data}

The experiment is carried out using 250 dermoscopy images which comprises of training and testing set [19]. The sample contains 150 training set images and 100 testing set images. The training set images are trained using GLCM, SURF for global and local texture feature extraction. The process of classification is performed using SVM and $\mathrm{KNN}$ and it classifies whether the test image provided is melanoma or non-melanoma. The experiments were carried out using the software Matlab R2015a. The sample input data images are given in the below Figure 2.

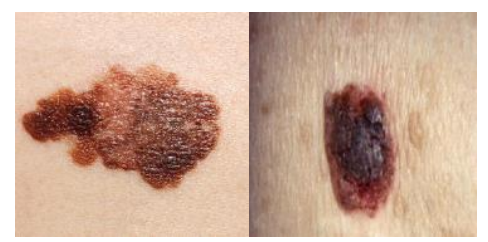

Figure 2. Sample Dermoscopy Images

\subsection{Experimental Results}

\subsubsection{Gray Level Co-occurence Matrix (GLCM)}

The dermoscopic images are taken as an input and the skin lesion is extracted from the image using Otsu's thresholding algorithm. The original and its segmented image is given in the below Figure 3 .

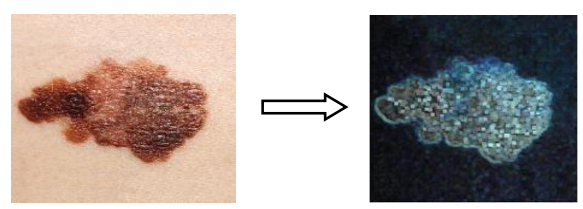

Figure 3. Original and Segmented Image

The global textural features are extracted using GLCM in the spatial domain and the statistical nature of texture is taken into consideration. A gray tone spatial dependency matrix is computed for a given image and a set of eight textural features are extracted from this matrix. The features described contain information about the image textural 
characteristics. These measures indicate the complexity and nature of gray tone transition present in an image. These eight textural features are given as an input to the SVM and KNN classifier. The texture features claculated in four different orientation angles is given in the below Table 1.

Table 1. Results of Texture Using GLCM

\begin{tabular}{|c|c|c|c|c|c|}
\hline & & \multicolumn{2}{|c|}{ Melanoma } & \multicolumn{2}{|c|}{ Non-Melanoma } \\
\hline & & Min & $\overline{\operatorname{Max}}$ & Min & $\operatorname{Max}$ \\
\hline \multirow{8}{*}{ Orientation $=0$} & Contrast & 0.44 & 0.49 & 0.27 & 0.47 \\
\hline & Correlation & 0.97 & 0.97 & 0.98 & 0.99 \\
\hline & Disimilarity & 0.16 & 0.21 & 0.06 & 0.1 \\
\hline & Energy & 0.32 & 0.4 & 0.44 & 0.51 \\
\hline & Entropy & 1.48 & 1.76 & 0.75 & 1.09 \\
\hline & Homogenity & 0.92 & 0.94 & 0.97 & 0.99 \\
\hline & Maximum Probability & 0.53 & 0.62 & 0.59 & 0.63 \\
\hline & Inverse diff moment & 0.99 & 0.99 & $\mathbf{1}$ & $\mathbf{1}$ \\
\hline \multirow{8}{*}{ Orientation $=45$} & Contrast & 7.13 & 9.14 & 4.25 & 7.97 \\
\hline & Correlation & 0.5 & 0.58 & 0.67 & 0.78 \\
\hline & Disimilarity & 1.49 & 1.63 & 0.67 & 1.15 \\
\hline & Energy & 0.19 & 0.23 & 0.3 & 0.44 \\
\hline & Entropy & 2.27 & 2.49 & 1.18 & 1.56 \\
\hline & Homogenity & 0.7 & 0.71 & 0.82 & 0.89 \\
\hline & Maximum Probability & 0.4 & 0.46 & 0.47 & 0.61 \\
\hline & Inverse diff moment & 0.91 & 0.92 & 0.93 & 0.96 \\
\hline \multirow{8}{*}{ Orientation $=90$} & Contrast & 13.84 & 16.9 & 8.35 & 17.01 \\
\hline & Correlation & 0.06 & 0.2 & $\mathbf{0 . 3}$ & 0.53 \\
\hline & Disimilarity & 2.66 & 2.87 & 1.26 & 2.44 \\
\hline & Energy & 0.1 & 0.13 & 0.21 & 0.4 \\
\hline & Entropy & 2.66 & 2.88 & 1.31 & 1.79 \\
\hline & Homogenity & 0.51 & 0.54 & 0.66 & 0.82 \\
\hline & Maximum Probability & 0.24 & 0.29 & $\mathbf{0 . 3 3}$ & 0.6 \\
\hline & Inverse diff moment & 0.84 & 0.85 & 0.85 & 0.93 \\
\hline \multirow{8}{*}{ Orientation $=135$} & Contrast & 14.64 & 17.1 & 8.78 & 19.57 \\
\hline & Correlation & 0.05 & 0.21 & 0.2 & 0.51 \\
\hline & Disimilarity & 2.7 & 3 & 1.33 & 2.8 \\
\hline & Energy & 0.1 & 0.12 & 0.21 & 0.38 \\
\hline & Entropy & 2.61 & 2.92 & 1.34 & 1.75 \\
\hline & Homogenity & 0.48 & 0.52 & 0.61 & 0.8 \\
\hline & Maximum Probability & 0.24 & 0.29 & 0.32 & 0.58 \\
\hline & Inverse diff moment & 0.83 & 0.85 & 0.83 & 0.92 \\
\hline
\end{tabular}

\subsubsection{Speed Up Robust Features (SURF)}

The interest points were generated and the feature vectors are given as an input to the classifiers. The interest point generation is shown in the below Figure 4.
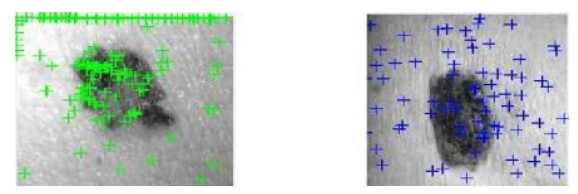

Figure 4. Sample of Generation of Interest Points 


\subsection{Performance Analysis}

\subsubsection{Performance Metrics}

The performance of the proposed system is evaluated on the basis of different metrics namely sensitivity, specificity, accuracy, precision and F1 score.

Sensitivity measures the true positive rate and it is given by

$$
\text { Sensitivity }=\frac{T P}{T P+F N}
$$

Specificity measures the true negative rate and it is given by

$$
\text { Specificity }=\frac{T N}{T N+F P}
$$

Accuracy is defined by

$$
\text { Accuracy }=\frac{T P+T N}{T P+F N+F P+T N}
$$

Precision is defined as the positive predictive value (PPV) and it is given by

$$
\text { Precision }=\frac{T P}{T P+F P}
$$

F1 score is the harmonic mean of precision and sensitivity and it is given by

$$
F 1 \text { Score }=\frac{2 T P}{2 T P+F P+F N}
$$

where TP-True Positive, TN-True Negative, FN-False Negative, FP-False Positive.

\subsubsection{Result Analysis}

The classification result shows that the accuracy of texture feature extraction using global texture extraction GLCM by SVM and KNN is $77 \%$ and $72 \%$. The accuracy of local feature extraction using SURF by SVM and KNN is $86 \%$ and $84 \%$ respectively. The experimental result shows that the texture feature extracted using SURF outperforms GLCM and SIFT in both the classifiers SVM and KNN. In the proposed system, SURF texture descriptor with SVM classifier provides better classification accuracy. For the input image data set taken for experimental analysis, local texture feature outperforms the global texture feature. The classification results are shown in the below Table 2 .

Table 2. Comparison of Feature Extraction Techniques GLCM, SIFT \& SURF by SVM, KNN

\begin{tabular}{|l|c|c|c|c|c|c|}
\hline \multirow{2}{*}{ Measures } & \multicolumn{2}{|c|}{ GLCM } & \multicolumn{2}{c|}{ SIFT } & \multicolumn{2}{c|}{ SURF } \\
\cline { 2 - 7 } & SVM & KNN & SVM & KNN & SVM & KNN \\
\hline Sensitivity & 77.9 & 72.4 & 80.1 & 77.7 & 86.2 & 84.1 \\
\hline Specificity & 78.4 & 76.5 & 82.4 & 83.5 & 88.4 & 86.5 \\
\hline Accuracy & 79.3 & 78.2 & 83.4 & 81.2 & 87.3 & 85.2 \\
\hline Precision & 77.1 & 76.4 & 79.9 & 78.4 & 78.9 & 77.4 \\
\hline F1 Score & 81.4 & 80.3 & 81.3 & 82.1 & 84.3 & 84.1 \\
\hline
\end{tabular}




\section{Conclusion}

A feature extraction model using global and local method was proposed for the identification of melanoma from dermoscopic images. The image is preprocessed and it is segmented using simple adaptive thresholding algorithm. Then the filtered image is subjected to feature extraction. These texture features are used to evaluate skin lesion discrimination using GLCM and SURF. The process of classification is done using SVMRBFand KNN. The experimental result shows that the local texture feature descriptor SURF along with SVM-RBF provides better classification results of sensitivity $86.2 \%$, specificity $88.4 \%$ and accuracy $87.3 \%$.

\section{References}

[1] "Cancer facts and figures 2012", American Cancer Society, Atlanta, GA, USA, Tech.Rep, (2012).

[2] DermoscopyTutorial, (2003), [online]. Available: http://www.dermoscopy.org/atlas/base.html

[3] M. Robert Haralick, K. Shanmugam, I. Dinstein., "Textural features for Image classification", IEEE Transactions on Systems, Man and Cybernatics, (1973), pp.610-621.

[4] D. Lowe, "Distinctive Image Features from Scale Invariant Keypoints", International Journal of Computer Vision, vol. 60, (2004), pp. 91-110.

[5] H. Bay, A. Ess, T. Tuytelaars, L. Van Gool, "Speeded-up robust features", Computer Vision and Image Understanding, vol.110, no.3, (2008), pp.346-359.

[6] H. Ganster, A. Pinz, E. Wilding, M. Binder, H. Kittler, "Automated melanoma recognition”, IEEE Trans.Med. Imaging, vol.20, no.3, (2001), pp. 233-239.

[7] M.E. Celebi, H.A. Kingravi, B. Uddin, H. Iyatomi, Y. Aslandogan, W. Stoecker, R. Moss, "A methodological approach to the classification of dermoscopy images", Science Direct, Computerized Medical Imaging and Graphics, vol. 31, no. 6, (2007), pp. 362-373.

[8] P. Rubegni, G. Cevenini, M. Burroni, R. Perotti, G. Dell'Eva, P. Sbano, C. Miracco, “Automated diagnosis of pigment skin lesions", International Journal of Cancer, vol. 101, no. 6, (2002), pp. 576-580.

[9] K. Mikolajczyk, C. Schmid, "A performance evaluation of local descriptors", PAMI 27, (2005), pp. $1615-1630$.

[10] K. Mikolajczyk, C. Schmid, "Indexing based on scale invariant interest points", ICCV, vol. 1, (2001), pp. 525- 531.

[11] D. Lowe, "Object recognition from local scale-invariant features", ICCV, (1999).

[12] C. Barata, M. Ruela, M. Francisco, T. Mendonca, J.orge S.Marques, "Two systems for the Detection of Melanomas in Dermoscopy Images Using Texture and Color Features”, IEEE systems Journal, vol.8, no.3, (2014).

[13] A. Omar, ,D. Buket Barkana, "Non invasive Real Time Automated skin Analysis system for Melanoma Early Detection and prevention", IEEE Journal of Translation Engineering in Health and Medicine, (2015).

[14] Di Leo,G., A. Paollillo, P. Sommella, C. Liguori, "An improved procedure for the automatic detection of dermoscopic structures in digital elm images of skin lesions", IEEE computer society, (2008), pp.190194.

[15] X. Yuan, Z. Yang, G. Zouridakis, "SVM based texture classification and application to early melanoma detection", Proc. IEEE EMBS International Conference, (2006), pp.4775-4778.

[16] N. Y. Khan, B. Mccane, G. Wyvill, "SIFT and SURF performance Evaluation against various image deformations on benchmark Dataset", International conference on Digital Image computing techniques and applications, (2011).

[17] www.supportvectormachines.org

[18] https://en.wikipedia.org/wiki/K-nearest_neighbors_algorithm

[19] http://homepages.inf.ed.ac.uk/rbf/DERMOFIT 
International Journal of Multimedia and Ubiquitous Engineering Vol.12, No.5 (2017) 